\title{
Anticuerpos antimitocondriales y anticuerpos contra antígenos extraíbles del núcleo en pacientes con cirrosis biliar primaria
}

\author{
Antimitochondrial antibodies and antibodies to extractable nuclear antigens in patients with primary \\ biliary cirrhosis
}

José Enrique Oliva Menacho ${ }^{1 a}$, Jose Arturo Oliva Candela ${ }^{1 b}$

\section{RESUMEN}

Objetivo: Determinar la frecuencia de anticuerpos antimitocondriales y de anticuerpos contra antígenos extraíbles del núcleo en pacientes con cirrosis biliar primaria. Material y métodos: Estudio de tipo cuantitativo, observacional y transversal, realizado en el Servicio de Inmunología del Hospital Nacional Arzobispo Loayza entre enero 2018 y marzo 2019. Se revisaron las historias clínicas de 30 pacientes con características presuntivas de cirrosis biliar primaria; para la detección de los anticuerpos antinucleares y anticuerpos antimitocondriales se empleó el kit inmunológico en sangre y observación con microscopio de inmunofluorescencia a 40X y para la detección de los anticuerpos contra antígenos extraíbles del núcleo se empleó el método Immunoblot. Resultados: Se estudiaron 30 pacientes con cirrosis biliar primaria, 20 fueron de sexo femenino $(66,7 \%)$. El patrón de tinción más frecuente fue el citoplasmático moteado reticular en $17(56,7 \%)$, seguido del patrón citoplasmático moteado reticular y patrón moteado en $7(23,3 \%)$ pacientes, y en menor frecuencia el patrón citoplasmático moteado reticular y patrón centromérico. Nueve $(42,9 \%)$ pacientes con cirrosis biliar primaria tenían anti-M2. Se demostró mayor frecuencia, $21(70 \%)$ de los pacientes con cirrosis biliar primaria tenían anticuerpos antimitocondriales. Conclusiones: Se encontró alta frecuencia de patrón citoplasmático moteado reticular en pacientes con cirrosis biliar primaria, se demostró asociación significativa con los anti-M2 y anticuerpos antimitocondriales.

PALABRAS CLAVE: Cirrosis hepática biliar primaria, anticuerpos, enfermedades mitocondriales, técnica del anticuerpo fluorescente indirecta, Perú. (Fuente: DeCS BIREME).

\section{SUMMARY}

Objective: To determine the frequency of antimitochondrial antibodies and antibodies to extractable nuclear antigens in patients with primary biliary cirrhosis. Methods: Cross-sectional study carried-out in the Servicio de Inmunología del Hospital Nacional Arzobispo Loayza from January 2018 to March 2019. Clinical charts of 30 patients with presumptive clinical features of primary biliary cirrhosis were reviewed. We used an immunologic blood kit and an immunofluorescence microscopy at $40 \mathrm{~K}$ to detect antinuclear antibodies and antimitochondrial antibodies, an immunoblot was used to detect antibodies to extractable nuclear antigens. Results: 30 patients were included in the study, 20 were female $(66.7 \%)$. The most frequent stain pattern was the cytoplasmic reticular mottled pattern; 17 (56.7\%) followed by the mottled pattern, 7 (23.3\%) and the least frequent pattern was the centromeric pattern. Nine patients $(42.9 \%)$ with primary biliary cirrhosis had anti-M2. Most of the patients with primary biliary

Hospital Nacional Arzobispo Loayza. Lima, Perú.

Magíster en Docencia e Investigación en Salud.

Biólogo en Inmunología. 
cirrhosis had antimitochondrial antibodies 21(70\%). Conclusions: We found a high frequency of the cytoplasmic reticular mottled pattern among patients with primary biliary cirrhosis and an association with anti-M2 and with antimitochondrial antibodies.

KEYWORDS: Primary biliary liver cirrhosis, antibodies, mitochondrial diseases, indirect fluorescent antibody technique, Peru. (Source: MeSH NLM).

\section{INTRODUCCIÓN}

La colangitis biliar primaria (CBP), inicialmente llamada cirrosis biliar primaria (1), es una enfermedad hepática inflamación y destrucción progresiva de los ductos biliares interlobulares, originando colestasis, fibrosis, cirrosis y enfermedad hepática terminal en pacientes no tratados $(2,3,4)$. Se cree que la etiología de la cirrosis biliar es una combinación de factores de riesgo genéticos y desencadenantes ambientales $(5,6,7)$.

La cirrosis biliar primaria se considera un modelo de enfermedad autoinmune sobre la base de varias características, incluyendo la presencia de una respuesta inmune directa y muy específica a los autoantígenos mitocondriales, predominancia femenina y homogeneidad entre los pacientes (8). A pesar del hecho de que los objetivos mitocondriales son proteínas omnipresentes expresadas en todas las células nucleadas, la inmunopatología de la CBP se carácteriza por la presencia de infiltrados de células T CD4 + y CD8 + en el hígado y la destrucción selectiva de células epiteliales biliares (BEC) $(9,10)$. Esto sugiere que las BEC pueden tener características inmunológicas únicas, si bien actualmente se acepta que los anticuerpos mitocondriales son los marcadores serológicos más específicos de la CBP $(11,12)$.

El anticuerpo anti mitocondrial es un autoanticuerpo altamente específico para la cirrosis biliar primaria (13), que se dirige al ácido lipoico presente en los complejos de 2-oxo-ácido deshidrogenasa ubicados en la membrana mitocondrial interna (14), además de una pérdida en la tolerancia humoral, hay un aumento de linfocitos autorreactivos de diferenciación CD $4+$ CD8 + piruvato deshidrogenasa complejo (PDC-E2)células T específicas en el hígado $(15,16)$.

Los anticuerpos antinucleares y los anticuerpos anti mitocondriales mediante inmunofluorescencia indirecta (IFI), en líneas celulares y tejido de riñón de rata, se considera la prueba inicial de laboratorio que apoya al diagnóstico de las enfermedades autoinmunes y enfermedades hepáticas debido a su alta sensibilidad (17).

Al presente, en la revisión bibliográfica, en el Perú no se reportan trabajos de investigación relacionados a la detección de anticuerpos antinucleares y anticuerpos antimitocondriales mediante inmunofluorescencia indirecta, en líneas celulares y tejido de riñon de rata. Este estudio contribuirá como ayuda en el diagnóstico de enfermedades de cirrosis biliar hepatica primaria. Beneficiará el diagnóstico de cirrosis biliar primaria según criterios inmunológicos laboratoriales del Colegio Americano de Reumatología.

El estudio tuvo como objetivo determinar la frecuencia de anticuerpos antimitocondriales y de anticuerpos contra antígenos extraíbles del núcleo en pacientes con cirrosis biliar primaria.

\section{MATERIAL Y MÉTODOS}

Estudio observacional, transversal realizado en el Servicio de Inmunología del Hospital Nacional Arzobispo Loayza entre enero 2018 y marzo 2019. Se revisaron las historias clínicas de 30 pacientes con diagnóstico presuntivo de cirrosis biliar primaria y muestras de suero de pacientes fueron procesados.

Los criterios de diagnóstico de cirrosis biliar primaria fueron: detección de anticuerpos antimitocondriales superior a 1:40, incremento de fosfatasa alcalina de 2 a 10 veces el valor normal durante más de 6 meses e histopatología compatible.

Criterios de inclusión: Pacientes con cirrosis biliar primaria, historia clínica completa, resultados de anticuerpos antinucleares por la técnica Inmunofluorescencia Indirecta, anticuerpos contra antígenos nucleares extraíbles por la técnica Inmunobloting y anticuerpos antimitocondriales por inmunofluorescencia, realizados entre enero 2018 y junio del 2019. 
Se excluyeron a los pacientes a quienes se determinó anticuerpos antinucleares y anticuerpos contra antígenos nucleares extraíbles por la técnica ELISA en pozo (Ensayo por Inmunoadsorción Ligado A Enzimas).

Para la detección de los patrones de tinción de anticuerpos antinucleares se empleó un kit comercial de procedencia Alemania, se realizó la dilución 1/160 para confirmar la positividad de anticuerpos antinucleares bajo observación con microscopio de inmunofluorescencia a 40X, se colocó un control positivo estandarizado en cada impronta, suero humano con anticuerpos antinucleares (ANA) patrón moteado grado IV, azida de sodio $0,95 \mathrm{~g} / \mathrm{l}$. La especificidad del control positivo ANA es verificada frente al suero de referencia $\mathrm{AF} / \mathrm{CDC} 1$ del Centers for Disease Control (CDC), Atlanta, USA y un control negativo estandarizado en cada impronta, que consistió en suero humano negativo a tamizaje infeccioso y ANA. Se numeraron las improntas en caso de correr más de 10 muestras y se procesaron rápidamente para evitar desecación de la impronta.

Para la detección de anticuerpos antimitocondriales se empleó el método IIFT: Kidney (Rat), un kit comercial de procedencia Alemania, se realizó la dilución 1/40,1/100 para la positividad y se observó con microscopio de inmunofluorescencia a 40X.

Para la detección de los anticuerpos contra antígenos extraíbles del núcleo en suero se empleó el método Immunoblot kit comercial de procedencia Lübeck, Alemania, y se colocó un control positivo estandarizado en la tira de reacción Immunoblot. El control positivo fue suero humano con anticuerpos anti-SSA(Ro), anti-SSB(La), anti-Sm, anti-Sm/ RNP, antiJo1 y anti-Scl70, azida de sodio $15 \mathrm{mmol} / \mathrm{l}$, calibrados frente al correspondiente suero de referencia ANA del CDC de Atlanta, USA y control negativo estandarizado, consistente en suero humano negativo a tamizaje infeccioso y ANA negativo.

\section{Análisis estadístico}

Se usó estadística descriptiva y el paquete estadístico SPSS v. 25 para Windows y el programa Microsoft Excel 2016. Se utilizó la prueba de Chi cuadrado de Pearson para las variables categóricas, considerando significativo un valor de $\mathrm{p}<0,05$.

Este estudio fue conducido de acuerdo con los principios éticos que tienen su origen en la Declaración de Helsinki, el estudio fue evaluado y aprobado por el Comité de ética del Instituto de Ética en Salud de la Universidad Nacional Mayor de San Marcos y por el del Hospital Nacional Arzobispo Loayza.

\section{RESULTADOS}

Se estudiaron treinta pacientes con cirrosis biliar primaria, veinte $(66,7 \%)$ del sexo femenino. Veintiuno (70\%) tenían cirrosis biliar primaria, tres $(10 \%) \mathrm{CBP}$ y CREST, cinco (16,7\%) CBP y S. Sjögren, y uno CBP y Lupus eritematoso sistémico (LES).

La frecuencia del patrón de tinción más frecuente fue del citoplasmático moteado reticular en diecisiete

Tabla 1. Patrones de tinción de anticuerpos antinucleares y enfermedades.

\begin{tabular}{lccccc}
\hline Enfermedad & CBP & $\begin{array}{c}\text { CBPy } \\
\text { CREST }\end{array}$ & $\begin{array}{c}\text { CBP y S. } \\
\text { Sjögren }\end{array}$ & $\begin{array}{c}\text { CBPy } \\
\text { LES }\end{array}$ & Total \\
\hline Patrón Citoplasmático Moteado Reticular & $16(76,2 \%)$ & $1(33,3 \%)$ & 0 & 0 & $17(56,7 \%)$ \\
$\begin{array}{l}\text { Patrón Citoplasmático Moteado Reticular y } \\
\text { Patrón Moteado }\end{array}$ & $3(14,3 \%)$ & 0 & $3(60,0 \%)$ & $1(100,0 \%)$ & $7(23,3 \%)$ \\
$\begin{array}{l}\text { Patrón Citoplasmático Moteado Reticular y } \\
\text { Patrón NUMA-1 Huso acromático }\end{array}$ & 0 & $1(33,3 \%)$ & $1(20,0 \%)$ & 0 & $2(6,7 \%)$ \\
$\begin{array}{l}\text { Patrón Citoplasmático Moteado Reticular y } \\
\text { Patrón Homogéneo }\end{array}$ & $2(9,5 \%)$ & 0 & $1(20,0 \%)$ & 0 & $3(10,0 \%)$ \\
$\begin{array}{l}\text { Patrón Citoplasmático Moteado Reticular y } \\
\text { Patrón centromérico }\end{array}$ & 0 & $1(33,3 \%)$ & 0 & 0 & $1(3,3 \%)$ \\
Total & 21 & 3 & 5 & 1 & 30 \\
\hline
\end{tabular}

CBP: Cirrosis biliar primaria; CREST: Esclerodermia limitada; LES: lupus eritematoso sistémico. 
Tabla 2. Anticuerpos contra antígenos extraíbles del núcleo y enfermedades.

\begin{tabular}{|c|c|c|c|c|c|}
\hline Enfermedad & CBP & $\begin{array}{c}\text { CBPy } \\
\text { CREST }\end{array}$ & $\begin{array}{l}\text { CBP y } \mathbf{S} . \\
\text { Sjögren }\end{array}$ & $\begin{array}{l}\text { CBP y } \\
\text { LES }\end{array}$ & Total \\
\hline Anti-Ro52, Anti-SSA, Anti-M2 & $2(9,5 \%)$ & 0 & 0 & 0 & $2(6,7 \%)$ \\
\hline Anti-Ro52, Anti-M2 & $5(23,8 \%)$ & 0 & $1(20,0 \%)$ & 0 & $6(20,0 \%)$ \\
\hline $\begin{array}{l}\text { Anti-Nucleosoma, Anti-Ro52, Anti-SSA, } \\
\text { Anti-M2 }\end{array}$ & $1(4,8 \%)$ & 0 & 0 & 0 & $1(3,3 \%)$ \\
\hline Anti-M2, Anti-CenpB & - & $2(66,7 \%)$ & 0 & 0 & $2(6,7 \%)$ \\
\hline Anti-M2 & $9(42,9 \%)$ & 0 & 0 & 0 & $9(30,0 \%)$ \\
\hline Anti-Histonas, Anti-M2 & $1(4,8 \%)$ & 0 & 0 & 0 & $1(3,3 \%)$ \\
\hline Anti-Nucleosomas, Anti-M2 & $1(4,8 \%)$ & 0 & 0 & 0 & $1(3,3 \%)$ \\
\hline $\begin{array}{l}\text { Anti-RO52, Anti-SSA, Anti-M2, Anti-SSB, } \\
\text { Anti-RNP/SM }\end{array}$ & 0 & 0 & $1(20,0 \%)$ & 0 & $1(3,3 \%)$ \\
\hline Anti-RIB p Protein, Anti-SSA, Anti-M2 & 0 & 0 & $1(20,0 \%)$ & 0 & $1(3,3 \%)$ \\
\hline Anti-Ro52, Anti-SSA, Anti-M2, Anti-SSB & 0 & 0 & $2(40,0 \%)$ & 0 & $2(6,7 \%)$ \\
\hline $\begin{array}{l}\text { Anti-Histonas, Anti-Nucleosomas, Anti-Rib p } \\
\text { protein, Anti-M2, Anti-RNP/SM, Anti-SM }\end{array}$ & 0 & 0 & 0 & $1(100,0 \%)$ & $1(3,3 \%)$ \\
\hline Anti-M2, Anti-SM & $2(9,5 \%)$ & 0 & 0 & 0 & $2(6,7 \%)$ \\
\hline Anti-Ro52, Anti-M2, Anti-CenpB, Anti-PM100 & 0 & $1(33,3 \%)$ & 0 & 0 & $1(3,3 \%)$ \\
\hline Total & 21 & 3 & 5 & 1 & 30 \\
\hline
\end{tabular}

CBP: Cirrosis biliar primaria; CREST: Calcinosis, fenómeno de Raynaud, alteraciones de la dismotilidad esofágica, esclerodactilia y telangiectasias; LES: Lupus Eritematoso sistémico.

(56,7\%), seguido del patrón citoplasmático moteado reticular y patrón moteado en siete $(23,3 \%)$ pacientes, tres pacientes tenían cirrosis biliar primaria, tres casos cirrosis biliar primaria y síndrome de Sjögren y uno cirrosis biliar primaria y LES (tabla 1).

Nueve $(42,9 \%)$ pacientes tuvieron anti-M2, cinco $(23,8 \%)$ anti-Ro52, anti-M2, mientras que otros pacientes tenían de dos a más los anticuerpos contra antígenos extraíbles del núcleo, la asociación de anticuerpos contra antígenos extraíbles del núcleo y enfermedades se encuentra distribuido en la tabla 2.

\section{DISCUSIÓN}

En los pacientes con cirrosis biliar primaria (CBP), los anticuerpos antinucleares están presentes en $30 \%$ a $50 \%(18,19)$. En la CBP, los anticuerpos antinucleares presentan reactividad específica manifestada por un patrón moteado nuclear o perinuclear circular (20).
El patrón moteado nuclear se encuentra en $17 \%$ de los pacientes con cirrosis biliar primaria (21), en la presente investigación se encontró que $76,2 \%$ de los pacientes tenían patrón citoplasmático moteado reticular y cirrosis biliar primaria. Los anticuerpos antimitocondriales son marcadores de diagnóstico para cirrosis biliar primaria y están dirigidos contra el complejo enzimático de las 2-oxo-ácido deshidrogenasas (22).

Los anticuerpos antimitocondriales (AMA) en los pacientes con cirrosis biliar primaria son los anticuerpos con mayor sensibilidad de todas las enfermedades hepáticas y su especificidad para distinguir pacientes con cirrosis biliar primaria de pacientes con hepatitis autoinmune es de $92 \%$ (23).

Los altos títulos de anticuerpos contra los elementos mitocondriales son característicos de la enfermedad 
(24). Walker et al., fueron los primeros en encontrar AMA en los sueros de los pacientes con cirrosis biliar primaria (25).

Los AMA son detectables en diferentes enfermedades; pero anti-M2, AMA son predominantemente a la cirrosis biliar primaria (26), similar en lo encontrado a Sánchez et al., (27). La asociación de cirrosis biliar primaria con otras enfermedades autoinmunes está descrita en la literatura y en $32 \%$ al $55 \%$ de los casos se encuentra al menos una enfermedad autoinmune asociada. Las enfermedades con una asociación estadísticamente significativa en ambas publicaciones son la enfermedad tiroidea autoinmune, el síndrome de Raynaud y el síndrome de Sjögren $(28,29)$ en la presente investigación se demostró la cirrosis biliar asociada en un $30 \%$ a enfermedades de CREST, síndrome de Sjögren y lupus eritematoso sistémico.

En conclusión, se encontró alta frecuencia de patrón citoplasmático moteado reticular en pacientes con cirrosis biliar primaria, se demostró asociación con los anti-M2 y anticuerpos antimitocondriales, $\mathrm{y}$ es necesario detectar los anticuerpos contra antígenos extraíbles del núcleo por Immunoblot para el diagnóstico de cirrosis biliar asociada a otras enfermedades del tejido conectivo. La detección en estadios iniciales de la enfermedad con estos métodos, retrasa la progresión, con lo que puede normalizar la supervivencia, sobre todo si se indica con ácido ursodeoxicólico (AUDC) es un tratamiento seguro, sin efectos tóxicos, que mejora los análisis, a veces incluso el prurito.

\section{Declaración de financiamiento y de conflictos de interés:}

El estudio fue financiado por los autores. Declaran no tener conflictos de interés.

\section{Contribuciones de autoría:}

JEOM: Participó en la concepción del estudio, la recolección de datos, interpretación de los datos, redacción del artículo, aprobación de la versión final del artículo. JAOC: Revisión crítica del artículo y aprobación de la versión final del artículo.

\section{Correspondencia:}

José Enrique Oliva Menacho.

Correo electrónico: jose.oliva.m@upch.pe .

Celular: (511) 990009733

\section{REFERENCIAS BIBLIOGRÁFICAS}

1. Beuers U, Gershwin ME, Gish RG, et al. Changing nomenclature for PBC: From "cirrhosis" to "cholangitis". Dig Liver Dis. 2015; 47(11):924926.

2. Carey EJ, Ali AH, Lindor KD. Primary biliary cirrhosis. Lancet. 2015; 386(10003):1565-1575.

3. Kaplan MM, Gershwin ME. Primary biliary cirrhosis. N Engl J Med. 2005; 353(12):1261-1273.

4. Selmi C, Bowlus CL, Gershwin ME, Coppel RL. Primary biliary cirrhosis. Lancet. 2011; 377(9777):1600-1609.

5. Selmi C, Mayo MJ, Bach N, et al. Primary biliary cirrhosis in monzygotic and dizygotic twins: Genetics, epigenetics, and environment. Gastroenterology. 2004; 127(2):485-492.

6. Shin S, Moh IH, Woo YS, et al. Evidence from a familial case suggests maternal inheritance of primary biliary cholangitis. World J Gastroenterol. 2017; 23(39):7191-7197.

7. Cheung AC, LaRusso NF, Gores GJ, Lazaridis $\mathrm{KN}$. Epigenetics in the primary biliary cholangitis and primary sclerosing cholangitis. Semin Liver Dis. 2017; 37(2):159-174.

8. Uibo R, Kisand K, Yang CY, Gershwin ME. Primary biliary cirrhosis: a multi-faced interactive disease involving genetics, environment and the immune response. Acta Pathol Microbiol Immunol Scand Pathol. 2012; 120(1):857-871.

9. Gershwin ME, Mackay IR. The causes of primary biliary cirrhosis: Convenient and inconvenient truths. Hepatology. 2008; 47(2):737-745.

10. Leung PS, Wang J, Naiyanetr P, et al. Environment and primary biliary cirrhosis: electrophilic drugs and the induction of AMA. J Autoimmun.2013; 41(1):79-86.

11. Hu CJ, Zhang FC, Li YZ, Zhang X. Primary biliary cirrhosis: what do autoantibodies tell us? World J Gastroenterol. 2010; 16(29):3616-3629.

12 Lleo A, Invernizzi P, Gao B, Podda M, Gershwin ME. Definition of human autoimmunity autoantibodies versus autoimmune disease. Autoimmun Rev. 2010; 9(5):A259-A266.

13. Gershwin ME, Mackay IR, Sturgess A, Coppel RL. Identification and specificity of a cDNA encoding the $70 \mathrm{kd}$ mitochondrial antigen recognized in primary biliary cirrhosis. J Immunol. 1987; 138(10):3525-3531.

14. Moteki S, Leung PS, Dickson ER, et al. Epitope mapping and reactivity of autoantibodies to the E2 component of 2-oxoglutarate dehydrogenase complex in primary biliary cirrhosis using 
recombinant 2-oxoglutarate dehydrogenase complex. Hepatology. 1996; 23(3):436-444.

15. Kita H, Matsumura S, He XS, et al. Quantitative and functional analysis of PDC-E2-specific autoreactive cytotoxic $\mathrm{T}$ lymphocytes in primary biliary cirrhosis. J Clin Invest. 2002; 109(9):1231- 1240.

16. Shimoda S, Van de Water J, Ansari A, et al. Identification and precursor frequency analysis of a common $\mathrm{T}$ cell epitope motif in mitochondrial autoantigens in primary biliary cirrhosis. J Clinical Invest. 1998; 102(10):1831-1840.

17. Delgado J, Palomino M, Rodríguez C. Protocolos de diagnóstico inmunológico en enfermedades autoinmunes. En Amengual J. Hepatopatías autoinmunes: cirrosis biliar primaria, hepatitis autoinmune y colangitis esclerosante primaria. Sociedad española de inmunología grupo español de autoinmunidad (GEAI). Elsevier, España. 2014. p.1-109.

18. Leung PSC, Coppel RL, Ansari A, Muñoz S, Gershwin M. Antimitochondrial antibodies in primary biliary cirrhosis. Semin Liver Dis. 1997; 17(1):61-69

19. Courvalin JC, Worman HJ. Nuclear envelope protein autoantibodies in primary biliary cirrhosis. Semin Liver Dis. 1997; 17(1):79-90.

20. Sternsdorf T, Guldner HH, Szostecki C, et al. Two nuclear dot-associated proteins, PML and Sp100, are often co-autoimmunogenic in patients with primary biliary cirrhosis. Scand J Immunol 1995; 42: 257-68.

21. Nakamura M, Kondo H, Mori T, et al. Anti-gp210 and anti-centromere antibodies are different risk factors for the progression of primary biliary cirrhosis. Hepatology. 2007; 45(1): 118-127.
22. Van de Water J, Cooper A, Surh CD, et al. Detection of autoantibodies to recombinant mitochondrial proteins in patients with primary biliary cirrhosis. N Engl J Med. 1989; 320(25):1377-1380.

23. Kenny RP, Czaja AJ, Ludwig J, Dickson ER. Frequency and significance of antimitochondrial antibodies in severe chronic active hepatitis. Dig Dis Sci. 1986; 31(7):705-711.

24. Kouroumalis E, Notas G. Pathogenesis of primary biliary cirrhosis: a unifying model. World J Gastroenterol. 2006; 12(15): 2320-2327.

25. Walker JG, Doniach D, Roitt IM, Sherlock S. Serological tests in diagnosis of primary biliary cirrhosis. Lancet 1965; 1(7390):827-831

26. Jiang XH, Zhong RQ, Yu SQ, Hu Y, Li WW, Kong XT. Construction and expression of a humanized M2 autoantigen trimer and its application in the diagnosis of primary biliary cirrhosis. World $\mathrm{J}$ Gastroenterol. 2003; 9(6):1352-1355.

27. Sánchez A, Hernández N, Chiodi D, Berrueta J, Robaina G, Pollio C, Mescia G. Cirrosis biliar primaria: Aspectos clínico epidemiológicos en una población uruguaya. Acta Gastroenterol Latinoam. 2013; 43(4):288-293.

28. Corpechot $\mathrm{C}$, Chrétien Y, Chazouillères O, Poupon R. Demographic, lifestyle, medical and familial factors associated with primary biliary cirrhosis. World J Hepatol. 2010; 53(1):162-169.

29. Gershwin M, Selmi C, Worman H, et al. USA PBC Epidemiology Group. Risk factors and comorbidities in primary biliary cirrhosis: a controlled interview-based study of 1032 patients. Hepatology. 2005; 42(5):1194-1202

Recibido: 02/04/2019

Aceptado: 19/04/2020 